\title{
Meaning of Hippocratic Oath in Modern Medicine and Medical Sciences
}

\section{Konrad Futyma}

2nd Department of Gynecology, Medical University of Lublin, Aleje Racławickie 1, 20-001 Lublin, Poland

\begin{abstract}
Hippocrates of Kos, the father of Western medicine, revolutionized medicine in ancient Greece. He gathered medical knowledge from other fields (magic, prophecy and philosophy) with which it had traditionally been associated and established it as a distinct discipline and profession. One of the doctrines he made, state that physicians must record their findings and investigative methods in a very clear and objective manner, so that these may be used by other physicians in order to constantly develop medical art [1]. And we are still doing it. We are constantly trying to show our own, sometimes slightly different and sometimes completely diverse, point of view of specific problem or condition. Through the history of medicine there is a lot of examples of great findings which at first, were subjects of ridiculation by the established authorities and societies, i.e. George Papanicolaou and his cervical smear which first met with skepticism and resistance from the medical community in 1928. This method was waiting for 15 years to be understood and used as a valid method for cervical cancer detection and saved numerous lives wide world [2]. We, as editors and reviewers, should consider any report of original research to be published which, of course, is supported by valid data with appropriate power. Even if those results are considered as strange, peculiar or even useless for actual medical knowledge. We should also give the priority to the papers describing novel diagnostic tools, markers and surgical methods over the sequential reports concerning the efficacy of some treatment options, especially when it was already confirmed on the large cohorts. New point of view may be important "trigger" for further development by other scientist or adjust it in other disciplines. Moreover, we have to remember that not every novelty or innovation must be confirmed in randomized controlled trials. This of course relates more to the diagnostic methods than treatment options. Nowadays, strong tendency to publish only results from the RCT can be observed. It is easy to understand that those results have the highest level of evidence because were obtained from properly designed research [3]. Evidence-based medicine is established on such results thus recommendations concerning certain medical problem can be made and professionals can rely on them in everyday clinical practice. It is built not only on the results of RCT but also on the diagnostic methods which, sometimes markedly, improve diagnosis accuracy. This of course influence on the therapy and its efficacy. We should not forget that the proper diagnosis is the key for success and we should not underestimate it.
\end{abstract}

This editorial is an appeal to the Editors and Reviewers who decide whether or not to publish someone's research just because it seems wired and useless. Sometimes, novel idea, which at glance looks peculiar, can be the base to create something more interesting and useful in our practice. I hope that this Journal will give this opportunity and will be perceived as open-minded for novel ideas from the gynecological field of interests. We are waiting for interesting ideas and propositions to develop our profession, even in better way that Hippocrates could think of. Do not stop in the research and don't feel discouraged if it will not be published at first submission. It might happen that next time the reviewer will understand this point of view and new discovery will contribute to medical profession improvement. First never follows.

\section{Publication History:}

Received: September 30, 2016

Accepted: February 08, 2017

Published: February 10, 2017

Keywords:

Hippocratic oath, Medical art, Medical sciences, Gynecology, Diagnosis

\section{Competing Interests}

The authors declare that they have no competing interests.

\section{References}

1. Garrison, FH (1966) History of Medicine. (4th edition), Philadelphia: W.B. Saunders Company, USA, $92 \mathrm{p}$.

2. Papanicolaou GN, Traut HF (1941) The diagnostic value of vaginal smears in carcinoma of the uterus. Am J Obstet Gynecol 42: 193.

3. Shekelle PG, Woolf SH, Eccles M, Grimshaw J (1999) Clinical guidelines: developing guidelines. BMJ 318: 593-596.
${ }^{*}$ Corresponding Author: Dr. Konrad Futyma, 2nd Department of Gynecology, Medical University of Lublin, Aleje Racławickie 1, 20-001 Lublin, Poland; E-mail: futymakonrad@mp.pl

Citation: Futyma K (2017) Meaning of Hippocratic Oath in Modern Medicine and Medical Sciences. Int J Gynecol Clin Pract 4: 127. doi: https://doi. org/10.15344/2394-4986/2017/127

Copyright: ( 2017 Futyma. This is an open-access article distributed under the terms of the Creative Commons Attribution License, which permits unrestricted use, distribution, and reproduction in any medium, provided the original author and source are credited. 